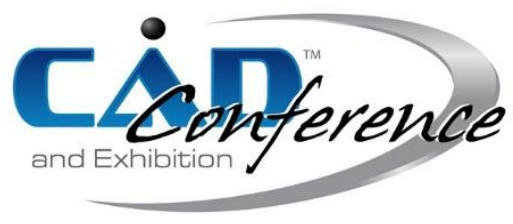

Title:

\title{
3D Modelling of the Knee Driven by Total Knee Arthroplasty Strategies
}

\author{
Authors: \\ Anna Ghidotti, anna.ghidotti@unibg.it, University of Bergamo \\ Andrea Vitali, andrea.vitali1@unibg.it, University of Bergamo \\ Daniele Regazzoni, daniele.regazzoni@unibg.it, University of Bergamo \\ Caterina Rizzi, caterina.rizzi@unibg.it, University of Bergamo \\ Keywords: \\ 3D Modelling, Total Knee Arthroplasty Planning, Customized Knee Prosthesis
}

DOI: 10.14733/cadconfP.2021.261-266

\section{Introduction:}

Total knee arthroplasty (TKA) is a surgical intervention, advised to treat knee osteoarthritis, which causes the progressive breakdown of the articular cartilage. Even if it is a very widely performed procedure, up to $25 \%$ of the patients are unsatisfied by the functional outcomes after surgery [4]. Implant malalignment seems to be one of the most important causes of surgical failures. Hence, in recent years, different technologies were implemented to reduce this risk: Computed Assisted Surgery (CAS) and Patient Specific Instrumentation (PSI) are the two most important ones. They are based on 3D knee modeling: 3D bone models are reconstructed, starting from computed tomography (CT) or magnetic resonance (MR) scans. These models can be used to build specific cutting blocks or to plan the intervention. The PSI guides are exploited to determine the level of resection, alignment, rotation, and size of the prosthesis components [4]. CAS involves the use of computer assistance to guide the surgeon to ensure accurate preparation of the femur and tibia before positioning the prosthesis. Planning for a TKA is crucial: it allows the surgeon to predict a range of implant sizes needed to be available in the operating room and provide him/her a reliable starting point in determining implant size and position without making aberrant cuts during the initial sizing. Most knee replacement prostheses are 'off-the-shelf', meaning they would be about the patient's size and a close fit, but not customized to the specific knee. In some cases, this implies an anatomical mismatch between the prosthetic components and the different people anatomies. Indeed, it is well known that there is anatomy difference between genders and among ethnicities, with the consequent possible improper fit of the implants [2]. In particular, two events can happen, both resulting in pain for the patient: overhang and underhang. The former occurs when the implant is bigger than the bone surface, which can impact on the soft tissues surrounding the knee joint; likewise, the latter occurs when the implant does not cover all the exposed cut bone. In order to improve the bone coverage, the implant fit and the range of motion, studies have been focused on the creation of customized implants. Thanks to the development in 3D imaging software and 3D printing technology, a further step can be made, moving from the virtual to the physical world. 3D printed models are useful for transferring information to the surgeon and testing the procedure on the patient-specific anatomy. Additive manufacturing techniques can be even exploited in order to realize patient-specific prosthesis. Using printing materials able to resemble the natural mechanical properties, the printed prosthesis can be ready to be implanted [1].

The current technologies, PSI, CAS and even robotic TKA that uses advanced surgical robots, are promising methods. However, conflict evidence is still reported in literature and more studies are needed to prove their superiority over conventional techniques in terms of clinical outcomes [3]. Even if there exist several commercial solutions, whose aim has always been to provide a reliable prosthesis with high 
survivorship, the most recent scientific literature is focusing on improving the patients' clinical outcome, such as kinematics and satisfaction. The investigation and the design of new methods and tools to replicate the native alignment and anatomy of the knee by preserving soft issues and ligaments are the areas of current and future developments in the field [3].

Main Aim:

This research work aims at providing a preliminary exploration of methods and tools to reduce the rate of patients' dissatisfaction and to design an implant with a shape like the native knee and a high level of detail. Starting from the analysis of different patients' knees, the first task is to define a procedure to perform an accurate and precise virtual planning of the intervention, to reach a personalized alignment for each patient by means of 3D modelling tools. The second purpose is to investigate possible procedures to create customized implants and overcome the limits of the standard prosthesis. This launch towards customization can be reached choosing the most suitable technique depending on the patient's characteristics. This means that the final goal is to provide a set of 3D modelling procedures among which the surgeons can choose the optimal one according to the patient's anatomy. To achieve the goals, free and open-source 3D modeling packages (i.e., 3D Slicer and Meshmixer) are exploited.

Method and Tools:

Medical images of fourteen different knees are used for the study. The images, obtained with a Magnetic Resonance (MR) technique, are analyzed together with the surgeons as well as each clinical case. Two open-source software tools are employed: 3D Slicer for the 3D modelling tasks and Meshmixer for the refinement of the mesh. In order to reconstruct the three-dimensional model of the knee, the segmentation process is done. Both manual and semiautomatic methods are used to obtain 3D models of the femur and tibia. In particular, for the manual segmentation, 'Draw' and 'Paint' tools are available in 3D Slicer; the former consists in surrounding the region of interest, while the latter in painting it. Instead, for the semi-automatic segmentation, 'Level tracing' is considered. It consists in adding uniform intensity region to selected segment. Once the bone models are ready, three procedures are studied and discussed with an orthopedic surgeon. The communication with the physicians is crucial to understand the feasibility of the proposed solutions. For five patients, models of the off-the-shelf prosthesis are available in six different sizes, obtained by a previous work through a 3D scanning procedure, using a mid-low cost and performant 3D laser scanner, i.e., NextEngine [6]. Hence, the bones are cut as in the traditional TKA, in order to fit the standard prosthesis. This technique allows to analyze the anatomy of the patient and plan the specific intervention. Then, two methods to overcome the limitations of the offthe-shelf implants are studied and customized prosthesis are created. In particular, the former consists of patient-specific resurfacing implants, which preserves the femoral bone, without any cut, and allows also choosing the thickness of the tibial component. The latter enables the creation of prosthesis that resemble the commercial ones but that are highly customizable. Fig. 1 lists the steps of each approach with the considered tools. The last step is the 3D printing of the models.

In the following the three procedures are presented and discussed.

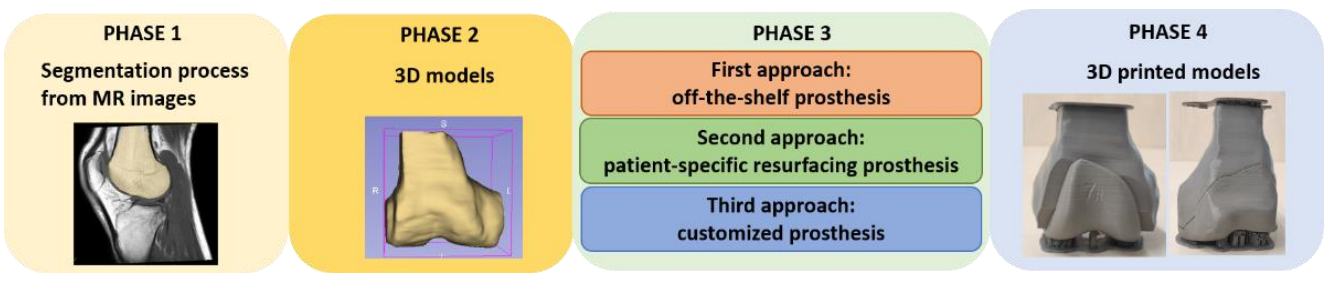

Fig. 1: Phases of the project, starting from MR images to the printed models.

\section{First Approach: Off-the-Shelf Prosthesis}

Standard off-the-shelf prosthesis models are available in six different sizes, from size 3 to size 8 . They are aligned to the bones, using 3D Slicer tools (Fig. 2). Firstly, the femoral components and tibial inserts are studied to understand the exact shape of the prosthesis, their thickness, the angles between the segments, the anterior/posterior (A/P) and medial/lateral (M/L) lengths. Then, even the A/P and M/L 
lengths of femur and tibia are measured (Fig. 2(a)). Once the correct femoral component size is identified, the prosthesis can be aligned to the segmented femur, following the three medical planes (Fig. 2(b)). In particular, the intercondylar notch and the trochlear surface are taken as reference. Some measurements can be made on the trochlea to exclude diseases, such as trochlear dysplasia, that can determine the instability of any implant used. In particular, it is interesting to analyze the sulcus angle, the lateral inclination angle (LTI) and the trochlear depth (TD). These measurements are reported in Fig.3. Moreover, attention is put not to create notching or patellar overstuff with the anterior cut. The position of the tibial insert is assessed in order to maximize tibial coverage, while avoiding overhang. Its correct rotation is crucial: it is typically centered on the junction between the medial and central third of the tibial tubercle. After the alignment, the bones are cut to fit the prosthesis. For this purpose, five planes are generated for cutting the femur, respectively for the anterior, posterior, distal, and the two slope surfaces (Fig. 2(c)). Only one cutting plane is enough for the tibia: $9 \mathrm{~mm}$ are easily cut from the bone in the less worn-out compartment, as suggested by the experienced surgeons and confirmed by the literature [5].

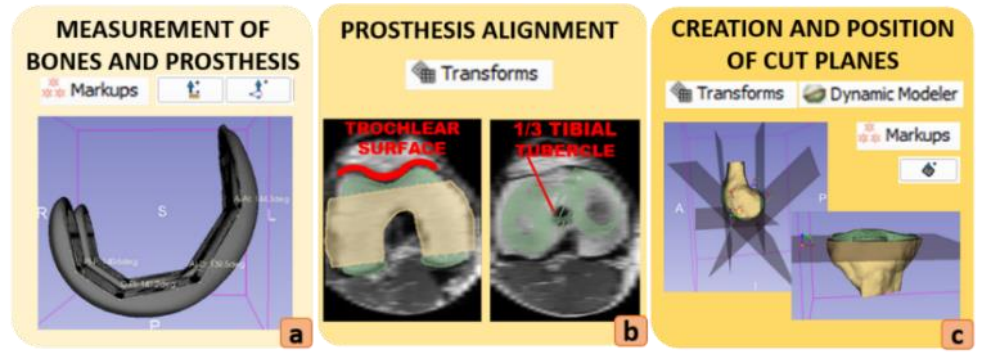

Fig. 2: Steps for the alignment of the off-the-shelf prosthesis to obtain the final result.
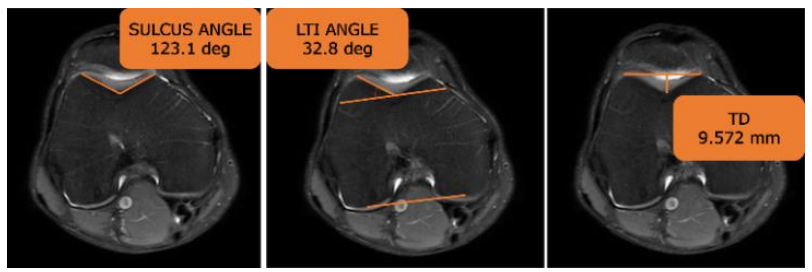

\begin{tabular}{|c|c|c|c|}
\hline Patient & $\begin{array}{c}\text { Sulcus } \\
\text { angle } \\
\text { (deg) }\end{array}$ & $\begin{array}{c}\text { LTI } \\
\text { angle } \\
\text { (deg) }\end{array}$ & $\begin{array}{c}\text { TD } \\
\text { (mm) }\end{array}$ \\
\hline $\mathbf{0 9}$ & 123.1 & 32.8 & 9.572 \\
\hline $\mathbf{1 4}$ & 130.8 & 21.8 & 7.517 \\
\hline $\mathbf{1 3}$ & 141.2 & 18.2 & 6.116 \\
\hline $\mathbf{0 7}$ & 131.1 & 27.8 & 8.032 \\
\hline $\mathbf{1 2}$ & 128.2 & 26.9 & 8.246 \\
\hline
\end{tabular}

Fig. 3: In the analyzed patients, sulcus angles are lower than $145^{\circ}$, LTI angles are greater than $11^{\circ}$ and TD lengths measure more than $3 \mathrm{~mm}$. These are the limits considered to exclude trochlear dysplasia.

\section{Second Approach: Patient-Specific Resurfacing Prosthesis}

To overcome the limits of conventional TKA, a more personalized approach is proposed. Starting from the bone segmentation, the personalized resurfacing prosthesis is created. Two different methods are used for the femoral and the tibial component, exploiting both 3D Slicer and Meshmixer tools (Fig. 4).

For the femoral component, it is observed that the articular cartilage follows the natural anatomy of the condyles. Hence, the inferior contour of the femur is followed in the sagittal plane: the points in which the cartilage starts and ends on the condyles are taken as reference for the prosthesis extremities. This first result is remodeled in order to make it more homogeneous. For the tibial component, a predetermined thickness is measured from the top of the tibia, in the less worn out compartment, in the coronal plane. A plan cut is used to divide the tibia in two parts; the upper part is remodeled in order to become the tibial component of the prosthesis. At this point, both components are aligned to the bones and they are ready to be printed.

Third Approach: Customized Prosthesis

A third approach, that is a compromise between the two previous ones, consists in the segmentation of both the femur and the femoral cartilage together. In case of severe worn of the cartilage, a fixed thickness is considered. 


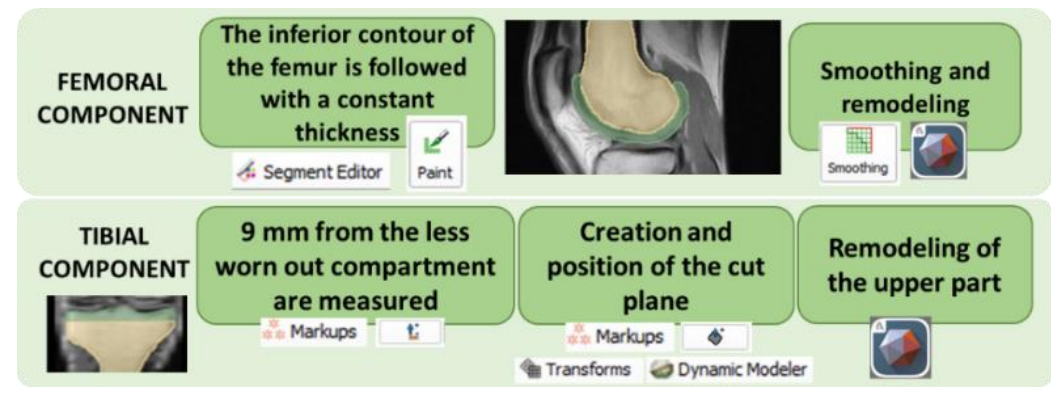

Fig. 4: Steps for the creation of patient-specific resurfacing prosthesis.

The unique segmentation is smoothed and remodeled (Fig. 5(a)). Then, cutting planes are created: five planes are added and located in the same positions and with the same angles, measured in the standard off-the-shelf prosthesis (Fig. 5(b)). In this way, it is possible to divide the model into two models: the cut femur and the customized prosthesis (Fig. 5(c)). Finally, the personalized femoral component is redefined in order to thin or thicker some parts and it is ready to be printed (Fig. 5(d)). The tibial component is realized in the same manner of the second approach to assure the complete coverage of the cut tibia. Even for this approach, 3D Slicer and Meshmixer tools are employed.
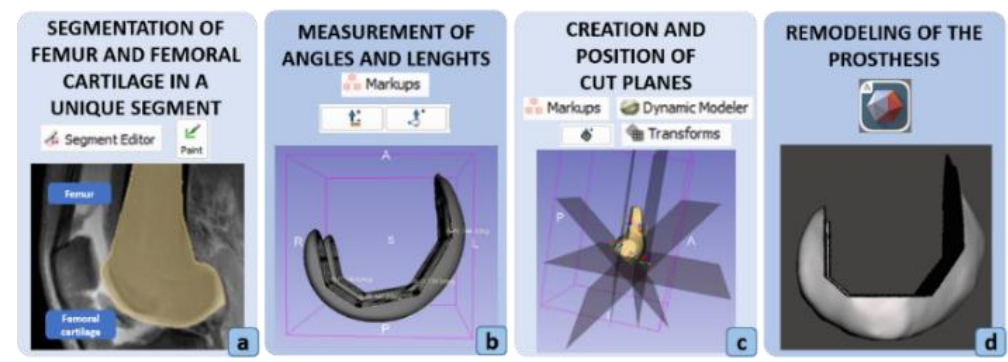

Fig. 5: Steps for the creation of a customized prosthesis.

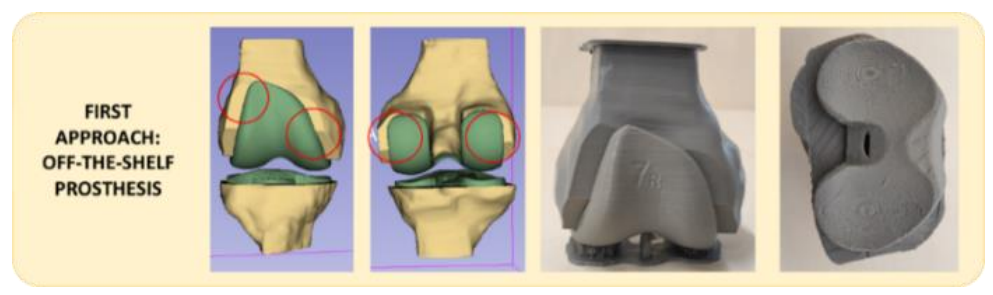

Fig. 6: Underhangs are highlighted with red circles in anterior and posterior views in the virtual environment. They are visible also in the 3D printed prototypes.

Results:

The accuracy of the 3D models, obtained through the segmentation process, depends on the quality of the MR exam and the thickness between the slices. The MR images, employed in the study, comes from the clinical practice, with a minimum thickness value of $1.65 \mathrm{~mm}$ and a mode value of $3.85 \mathrm{~mm}$. Even if it is not the optimal condition, it is possible to obtain acceptable results. Starting from the reconstructions, three procedures are proposed. Each time, the most suitable technique is chosen according to the patient's anatomy, knee size and articular cartilage damage. The planning of the intervention, obtained with the first approach, allows the surgeon to predict the size of the off-the-shelf 
prosthesis before the intervention. The insertion of the ideal size is crucial for the restoration of the correct biomechanics in the knee; otherwise, the range of motion may be reduced. In some cases, the implants do not perfectly fit the patient's anatomy. In particular, underhangs are noticed several times, when the implant does not cover all the exposed cut bone, with consequent pain in patients (Fig. 6).

Therefore, a more customizable solution is tried. With the second approach, it is possible to create a femoral component that perfectly fits the patient's anatomy, preserving the bone, without the need of any cut (Fig. 7(a)). The thickness of the implant can be chosen according to the available space between the femur and tibia, which is different from patient to patient. Furthermore, it is possible to select different thickness of the tibial component according to the need. In this way, a complete coverage of the cut tibia is guaranteed. However, it is known that osteoarthritis leads to the articular cartilage breakdown and can even affect the underlying bone. In the cases in which the bone is too much ruined, the prosthesis is not able to perfectly match it. Hence, a third approach is proposed, starting from the two previous techniques (Fig. 7(b)). It allows to create something that resembles the commercial off-theshelf femoral component, but highly patient-specific. It perfectly fits the patient's anatomy; there are no overhangs or underhangs and it matches the condylar offset and the intercondylar notch. The natural joint line is preserved by restoring the distal femoral offset. Moreover, there are no problems if the femur is too big or too small because the prosthesis follows the patient's femur. According to the surgeon, this solution is a good compromise between the previous two approaches. The strong point is its adaptability in presence or without the cartilage. Moreover, there are five cut planes as in the standard off-the-shelf prosthesis, that could be useful when even the bone is ruined.

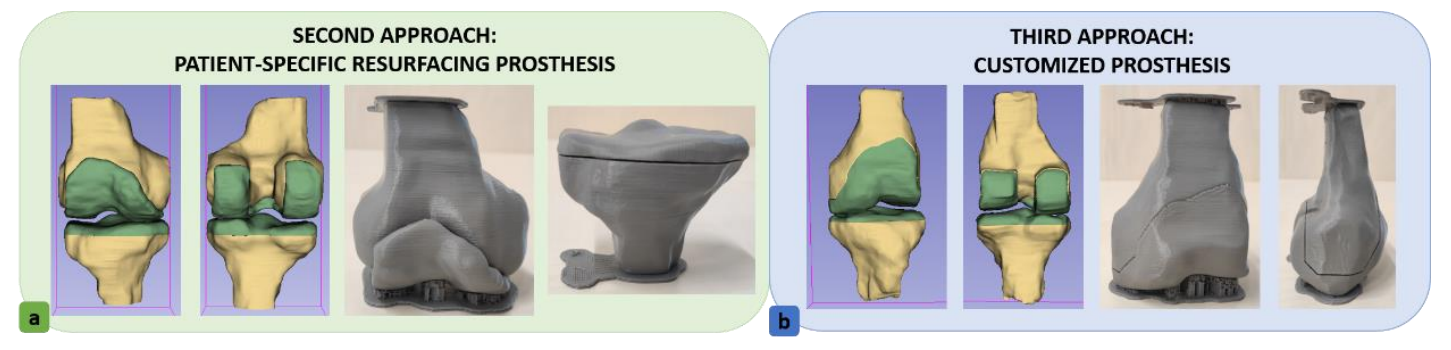

Fig. 7: A perfect match between the bone and the prosthesis in the second and third approach.

\section{Conclusions:}

The current work investigates methods and tools to replicate the native alignment and anatomy of the knee, which can be applied in the research field both for the surgical planning and the creation of patientspecific implants. After the reconstruction of the 3D model of the knee in the virtual space, it would be possible to study the patient's anatomy. Hence, depending on it, the suitable approach could be chosen, among the three previous procedures. If the patient has a quite standard anatomy, a planning with an off-the-shelf prosthesis could be performed. In more complex cases, it would be possible to choose between the last two approaches. If osteoarthritis has not affected the bone yet, it would be possible to preserve the bone and apply a patient-specific resurfacing implant. Otherwise, the customized prosthesis of the third approach is the one recommended. According to this preliminary study, each patient would receive the most suitable treatment, trying to improve their rate of satisfaction. The future work will be the automatization of the steps, presented in this research. In the meanwhile, a Finite Element analysis will be performed to understand the feasibility of the proposed solutions.

Acknowledgements:

The authors would like to thank Doctor Francesca De Caro for her precious clinical contribution.

References:

[1] Auricchio, F; Marconi, S.: 3D printing: clinical applications in orthopaedics and traumatology. EFORT

Proceedings of CAD’21, Barcelona, Spain, July 5-7, 2021, 261-266

(c) 2021 CAD Solutions, LLC, http://www.cad-conference.net 
Open Reviews Vol. 1, No. 5 (2016). https://doi.org/10.1302/2058-5241.1.000012. [2] Benazzo, F.; Ghiara, M.; Rossi, S.M.P. et al.: Clinical and radiological analysis of a personalized total knee arthroplasty system design. International Orthopaedics (SICOT) 43, 1113-1121 (2019). https://doi.org/10.1007/s00264-018-4095-4.

[3] Blakeney W.G.; Vendittoli PA. (2020) The Future of TKA. In: Rivière C., Vendittoli PA. Personalized Hip and Knee Joint Replacement. Springer, Cham. https://doi.org/10.1007/978-3-030-24243-5_15. [4] Giannotti, S.; Sacchetti, F.; Cittarelli, C. et al. Single-use, patient-specific instrumentation technology in knee arthroplasty: a comparative study between standard instrumentation and PSI efficiency system. Musculoskeletal Surg 104, 195-200 (2020). https://doi.org/10.1007/s12306-019-00612-3. [5] Murgier J.; Clatworthy M.: Variable rotation of the femur does not affect outcome with patient-specific alignment navigated balanced TKA, Knee Surgery, Sports Traumatology, Arthroscopy (2020) https://doi.org/10.1007/s00167-020-06226-8.

[6] Rossoni, M.; Regazzoni, D.; Vitali, A. et al. Knee prostheses reverse engineering: a preliminary investigation. Proceedings of the ASME 2020 IDETC-CIE Conference. Volume 9: 40th Computers and Information in Engineering Conference (CIE). https://doi.org/10.1115/DETC2020-22485. 